\title{
The Key Technology of Medical Image Cloud Service Based on "Internet + Medical Combination"
}

\author{
Xuan Zheng ${ }^{1}$, Lijuan Zheng ${ }^{2}$ \\ ${ }^{1}$ College of Information Engineering, Gannan Medical University, Ganzhou, Jiangxi, 341000, China \\ ${ }^{2}$ Collegel of Nursing, Gannan Medical University, Ganzhou, Jiangxi, 341000, China
}

Keywords: Internet, Medical conjoined, Medical image, Cloud services

\begin{abstract}
The rapid development of Internet and cloud computing technology provides an effective way to build a medical image service platform with low cost, high availability and high performance. The development of medical image cloud service technology and the construction of medical image sharing platform will help to integrate image equipment and expert resources, carry out medical image remote consultation and inquire about typical cases, which is of great significance to balance the medical resources and improve the diagnosis and treatment of the grass roots hospitals. Based on the author's learning and practical experience, this paper firstly analyzed the necessary conditions for the emergence of medical image cloud service, and then discussed the key technologies in medical image cloud service.
\end{abstract}

\section{Necessary conditions for the emergence of medical image cloud services}

Imaging diagnosis is very difficult and is urgently needed in primary hospitals. Medical image collaboration can fully share the image equipment of small and medium-sized hospitals and large hospitals expert talent resources. At the same time, through remote diagnosis assistance and teaching, it can also greatly improve the level of diagnosis and treatment of doctors in primary hospitals, so it can improve the quality of medical services.

Sharing can significantly reduce the medical costs. Remote consultation and referral can achieve the sharing of imaging equipment and talent resources, on the one hand, it can reduce the cost of introducing high-end imaging equipment and excellent image diagnosis experts. On the other hand, it can reduce the patient's repeated examination, and reduce the hospital's medical cost and the patient's medical expense. The technical standard is mature.

The international standards of medical image have been very mature. Since the release of the relevant standards, the framework is stable and widely used, and there is no conflict or competition in the field of medical image. At present, it has become the universal standard of medical image equipment and system.

\section{Analysis on key technologies of medical image cloud service}

\subsection{Overall structure}

The medical image post-processing cloud platform architecture diagram is shown in figure 1. For medical image post-processing cloud platform design, we should follow the principles of openness, transparency, high performance-price ratio, safety and reliability, etc. The medical image post-processing cloud platform follows the SOA technology architecture and is divided into three levels: Iaa S, Paa S and Saa S, which are infrastructure as a service, platform as a service and software as a service. The Iaa S layer is mainly the cloud storage DICOM gateway of medical image, DICOM standard exchange and routing layer and image cloud computing layer. The Paa S layer includes the image post-processing template library, which instantiates and packages the image post-processing to meet the different needs of thin and fat clients. Saa S layer is mainly responsible for cooperating with portal processing, to simplify workflow process and improve workflow efficiency. 


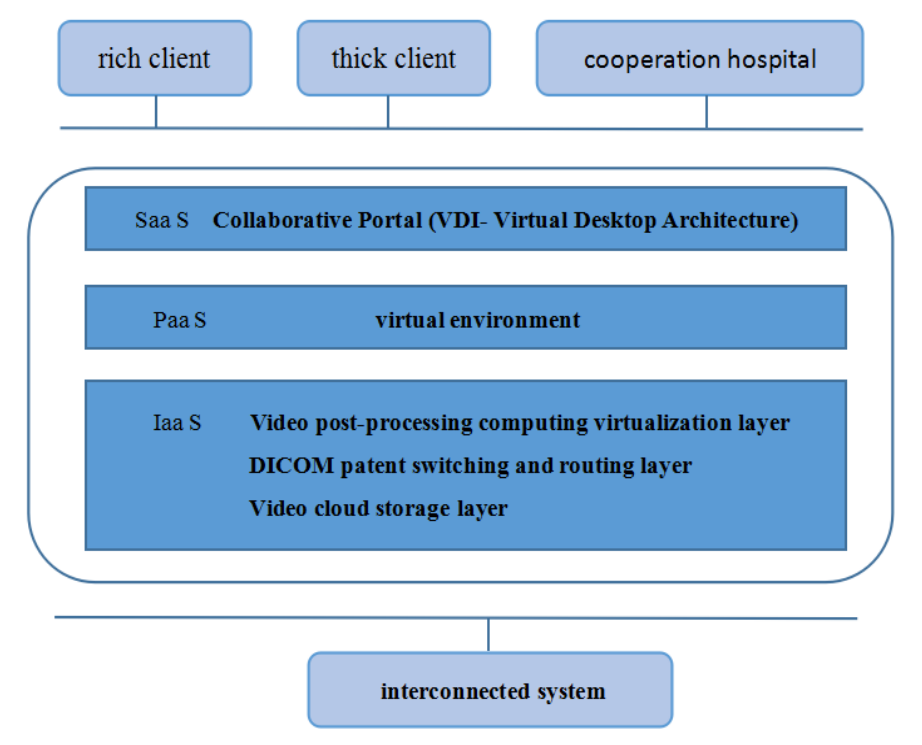

Fig 1. The medical image post-processing cloud platform architecture.

Medical image post-processing cloud platform can enable user countries to register data sets. For example, the communication data set of digital medical image and the data set of electropathic history are applied to the cloud platform of medical image post-processing, which lays a good foundation for sustainable development such as docking, data mining and medical quality control. The information flow of video post-processing cloud platform is divided into two parts: data storage process and reconstruction process. The specific flow information is as follows: 1) video data storage process: add DICOM remote node and associate with thin layer sequence on video equipment CTMR etc. The automatic routing function of PACS archived video and thin layer sequence is realized. Reconstruction process: the client puts forward the post-processing request of the video, and the medical image post-processing cloud platform carries on the message transmission. The video post-processing template is directly collected from the video post-processing template library and instantiated by the template library to realize video post-processing. Finally, the video post-processing results are pushed to the client by VDI technology.

\subsection{Cloud computing layer}

The cloud computing layer of medical image post-processing cloud platform provides an efficient, fast and flexible image post-processing computing for medical image post-processing cloud platform, and realizes the rapid reconstruction formedical image. In the cloud computing layer, the image post-processing applications are deployed in the virtual machine server to integrate various image post-processing software such as heart, blood vessel, nerve, tumor and so on, so as to realize the openness of the platform. At the same time, according to the number of concurrent virtual users, cloud computing layer can allocate hardware resources in time bu using GPU, CPU, MEM and other hardware clusters, so as to quickly achieve video reconstruction, push the results to the client to meet the individual video diagnosis and treatment. The entire physical topology is shown in figure 2. In the computing process of video post-processing platform, the virtualization technology Hyper-V is used to build the user desktop virtual machine quickly. Virtualization technology includes fast unified creation of desktop virtual machines, deployment of medical image post-processing applications, configuration of virtual machine templates, construction of virtual machine libraries, integration of video reconstruction template libraries, and rapid realization of video instantiation. Then, the virtual machine is allocated to individual or certain departments. When the configuration of the computer used by the medical staff is consistent, a pool of virtual machines with the same configuration can be established, and then the pool of virtual machines can be allocated to these users, and the virtual machines accessed by the user are dynamically allocated. Users can gain seamless access experience and realize flexible and dynamic virtual desktop 
technology, which is called "Virtual Desktop Architecture-VDI" technology.

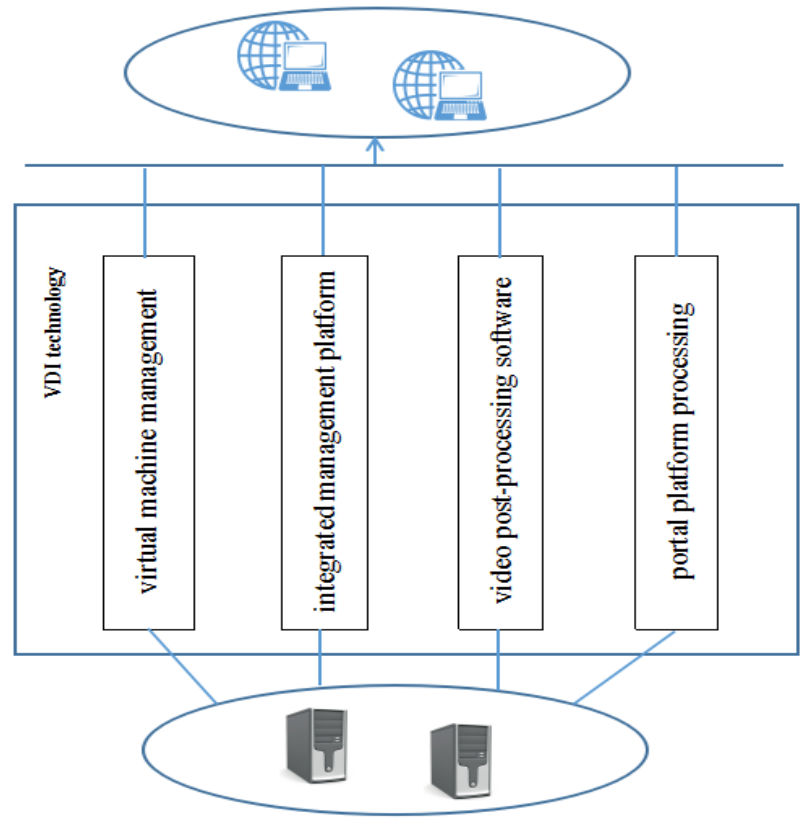

Fig 2. Physical topology diagram of cloud computing layer.

\subsection{Cloud storage layer}

Cloud storage is a new type of storage system with cloud structure, which is composed of multiple storage devices. Through cluster applications, distributed file systems or similar grid technology, a large number of different types of storage devices in the network can work together through application software, to provide data storage and business access services through a certain application software or interface. Cloud storage layer is the foundation of cloud computing layer and the core part of medical image post-processing cloud platform. In order to realize the fast storage of mass video data and meet the requirements of fast, efficient and flexible medical image post-processing, cloud storage system used in medical image post-processing cloud platform. The cloud storage system adopts the advanced asymmetric distributed architecture, which accords with the extension idea of scaleout which is popular in the industry at present. To make the system lead to the actual demand and guide the business development to the right direction has became the driving force of business innovation. The storage architecture of cloud computing is shown in figure 3.

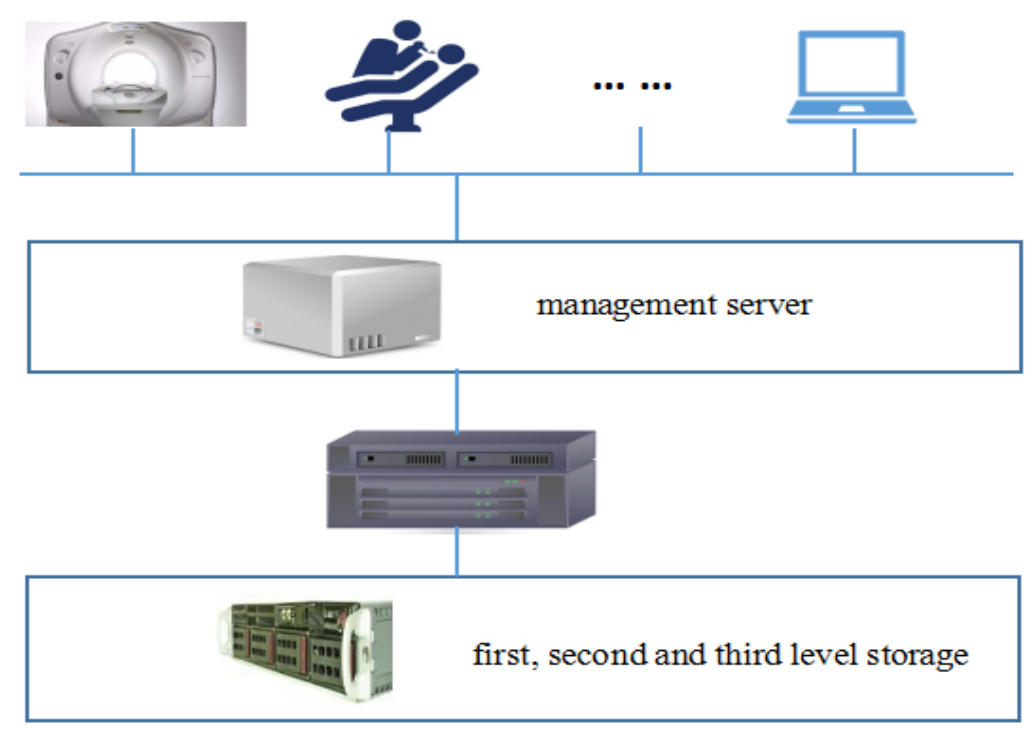

Fig 3. Cloud storage architecture of medical image. 
Integrated platforms and portals. As can be seen from the architecture diagram of the medical image post-processing cloud platform (figure 4), the entire medical image post processing integrated platform is associated with many systems. Through MPI, the medical image post-processing cloud integration platform establishes mapping relationship with other medical information systems, so the information exchange and interconnection of medical information systems are realized, and it is convenient for medical image post-processing cloud platform to realize information interaction and video collaborative therapy. The associated systems include EMR, LIS, PACS, HIS, TPS, information system, sports and recreation system, and personal health file system. The whole platform adopts DICOM standard, HL7 standard and IHE standard in the process of integration, which lays a solid foundation for the platform's sustainable development and later expansion or integration with different standards or systems. The video post-processing cloud platform based on cloud computing forms a complete portal platform through workflow model. It is the foundation and basic environment of workflow operation and provides workflow customization function. This platform can be used to customize the specific workflow required by the business, which can be executed in a secure and orderly manner on the service management platform. The service management platform model consists of workflow engine, script engine, message engine, SMS engine, event reminder, process monitoring, data service, security management, privilege control, layout management, personalized support. Medical image post-processing cloud platform involves many roles, departments, multiple platforms of collaborative operation. Constructing a collaborative portal platform for medical image post-processing cloud platform is of great significance for improving the overall performance of the platform.

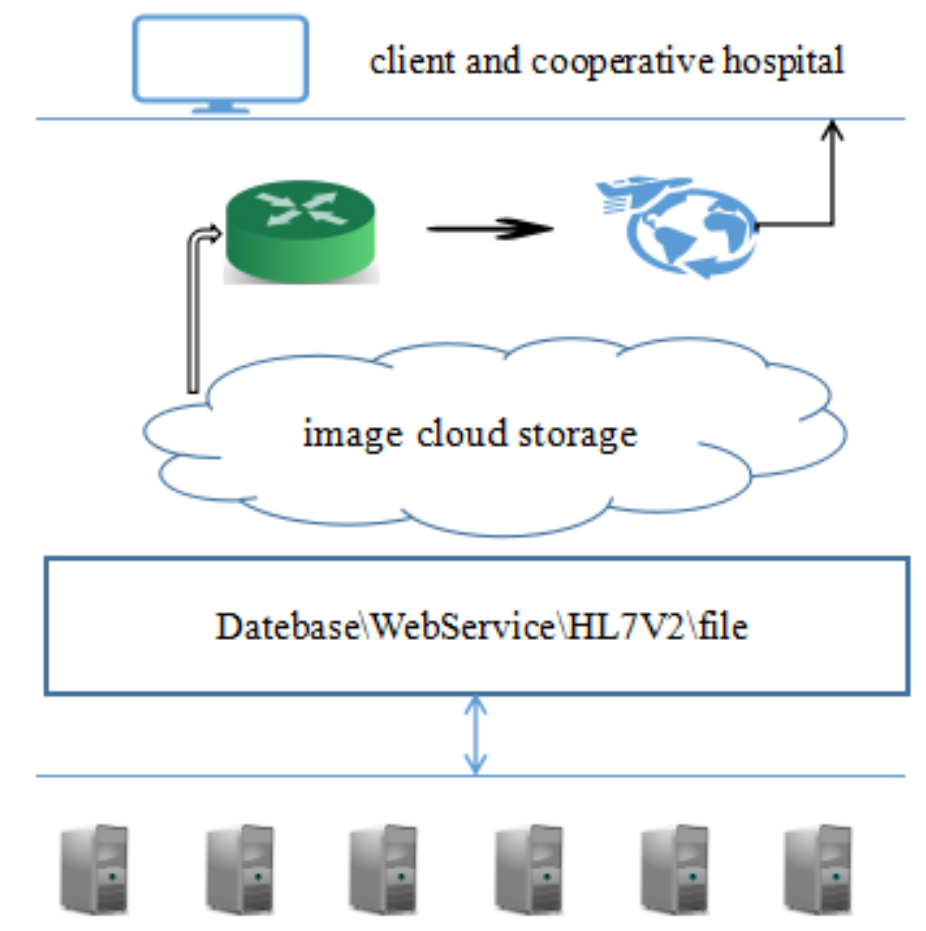

Fig 4. Integrated architecture diagram of post-processing platform.

\section{Conclusions}

Building cloud platform for medical image post-processing based on cloud computing has great significance for the later development of medical image remote consultation, video referral, virtual video specialist, distance learning, image content retrieval. To achieve the full sharing and efficient cooperation of video equipment and video diagnosis technology will help to balance medical resources, improve the level of diagnosis and treatment in grass-roots hospitals, improve the efficiency of video equipment use, and improve the quality of medical services. Reduce medical costs 


\section{Acknowledgements}

Project funds: humanistic subject of colleges and universities in Jiangxi Province - Research on the Application of Mobile Medical and Health Service for Medical big data processing (JC162005).

\section{References}

[1] Li Tiantian, Ma Rui, Qian Yajun. Practice of medical image Cloud platform based on Medical Association [J]. Chinese Digital Medicine, 2017, (11): 8-10+17.

[2] Wang Wei. A cloud processing method for medical image [P]. Jiangsu: CN106485079A 2017-03-08.

[3] Huang Xin, Shen Xiangbai. PACS medical image secure Transmission system based on Cloud Storage [J]. Aerospace Medicine and Medical Engineering, 2015, (05): 366-369.

[4] Wu Fuli, Zhang Tong, Liang Ronghua. Visual interactive platform for medical image based on cloud platform [J]. Chinese Science: Information Science, 2014, (11): 1432-1444. 